\title{
Efficient Distributed Joint Path Selection and Resource Allocation in Non-Cooperative Wireless Relay Networks
}

\author{
Vincenzo Lottici and Filippo Giannetti* \\ Dipartimento di Ingegneria dell'Informazione, Università di Pisa, Italia
}

\begin{abstract}
This paper proposes a distributed approach based on the network formation game (NFG) framework to joint path selection and resource allocation for a noncooperative multi-hop coded multicarrier relay network under outdated and imperfect channel state information. Simulation results corroborate the effectiveness of the proposed strategies, which offer goodput performance very close to the benchmark centralized case even with a considerable reduction in signalling and computational complexity.
\end{abstract}

\section{Introduction}

Long term evolution advanced (LTE-Advanced), IEEE 802.16j WiMAX and 5G networks can properly capitalize on multi-hop $(\mathrm{MH})$ relaying to significantly improve coverage, energy efficiency and battery life of devices [1]. According to the $\mathrm{MH}$ approach, one or more relay nodes (RNs) forward information between a source node (SN) and a destination node (DN), either in cooperative or non-cooperative [2] mode. Cooperative techniques are more energy efficient than non-cooperative ones, always providing a smaller outage probability, tough at the price of increasing processing and signaling traffic over the feedback channel. On the other side, in non-cooperative transmissions, a SN transmits its information passing through one or more RNs, called path, while the DN receives the signal only from one $\mathrm{RN}$, discarding emissions from other transmitters [2]. Hence, processing and signaling traffic is reduced and less widespread knowledge of channel state information (CSI) can be obtained, thus achieving more efficient and appealing solutions. In non-cooperative relaying, however, the path selection (PS) problem is a critical task. Additionally, advanced resource allocation (RA) techniques are required for an efficient use of radio resources among the nodes. In this context, network formation game (NFG) stand for an effective tool for the design of distributed PS algorithms [3]. The problem of MH relay selection is studied in [4] based on NFG for cognitive radio networks (see the references therein), while in [5] the network architecture for connecting RNs and SN to the DN is tackled by proposing an effective distributed PS algorithm, but a single carrier transmission is implemented and no RA strategy is considered.

\section{Contributions:}

1. The proposed joint PS and RA strategy represents a novel solution to optimize the goodput (GP) metric, i.e., the number of information bits delivered in errorfree packets per unit of time, for systems employing coded multicarrier schemes under imperfect and outdated CSI (due to non ideal estimation at the receiver and delay over the feedback channel, respectively);

2. The centralized solution is first discussed, pointing out its unfeasible computational complexity. Then, resorting to the NFG framework, the PS and RA problem is solved through a distributed algorithm, which significantly reduces the signalling traffic among nodes.

3. Further, exploiting the modified Bellman-Ford algorithm, a variant of the distributed NFG-based algorithm is illustrated, which allows to decrease the computational load for each node from combinatorial to a polynomial one.

4. Simulation results corroborate the effectiveness of the distributed strategies, offering GP performance very close to the benchmark centralized case, but with a considerable reduction of both signalling and computational complexity.

\section{System Model}

The multi-hop wireless network is composed by a $\mathrm{SN}, \quad \mathrm{D} \mathrm{DN}$ and $M \mathrm{RNs} \mathrm{R}_{i}, \quad i=1, \cdots, M$ (either fixed or mobile), where the latter decode and reencode the received signal according to the decodeand-forward (DF) scheme. Thus, SN sends data messages to the DN exploiting either a direct link or a multi-hop path with $L \leq M$ RNs. Let us define i) $\mathcal{P}_{\mathrm{S}}\left(\mathrm{SN}, \mathrm{R}_{i_{1}}, \cdots, \mathrm{R}_{i_{L}}, \mathrm{DN}\right) \in \mathcal{G}_{\mathrm{S}}$ as the path connecting $\mathrm{SN}$ to $\mathrm{DN}$ through the relays $\mathrm{R}_{i_{1}}, \ldots, \mathrm{R}_{i_{L}}$, where $\mathcal{G}_{\mathrm{S}}$ is the set of all possible paths between SN and DN, $0 \leq L \leq M, i_{1}, \cdots, i_{L} \in\{1, \cdots, M\}$, with $i_{j} \neq i_{k}$, $\forall j \neq k \in\{1, \cdots, L\}$ and $\mathrm{R}_{i_{L}} \triangleq \mathrm{DN}$ if $L=0$, i.e., the direct link SN-DN; ii) $l \in \mathcal{L} \triangleq\{0, \cdots L\}$ as the index of the generic link, where $l=0$ is the link SN-R $i_{i_{1}}$, $l=L$ is the link $\mathrm{R}_{i_{L}}$-DN and $0<l<L$ is the link $\mathrm{R}_{i_{l}}-\mathrm{R}_{i_{l+1}}$. A sequence of packets are transmitted exploiting a BIC-OFDM modulation with $N$ subcarriers within a bandwidth $B$. After encoding with rate $r \in \mathcal{D}_{\mathrm{r}}$, where $\mathcal{D}_{\mathrm{r}}$ is the set of possible code rates, 
the coded sequence is randomly interleaved. Then, the coded bits are Gray-mapped into $N_{\mathrm{s}}$ complexvalued symbols $\mathbf{x}_{l} \triangleq\left[x_{l, 1}, \cdots, x_{l, N}\right]^{\mathrm{T}}$, where $x_{l, n}$ is a $2^{m_{l, n}-\mathrm{Q} A M}$ unitary-energy symbol, $m_{l, n} \in \mathcal{D}_{\mathrm{m}} \triangleq$ $\left\{0,2,4, \cdots, m_{\max }\right\}$ being the number of bits allocated on the $n$th subcarrier and belonging to the bit loading (BL) vector $\mathbf{m}_{l} \triangleq\left[m_{l, 1}, \cdots, m_{l, N}\right]^{\mathrm{T}}$. Further, $\mathbf{x}_{l}$ is element-wise multiplied by the square root of the power allocation $(\mathrm{PA})$ vector $\mathbf{p}_{l} \triangleq\left[p_{l, 1}, \cdots, p_{l, N}\right]^{\mathrm{T}}$ satisfying $\sum_{n=1}^{N} p_{l, n} \triangleq P_{\text {tot }}$, where $P_{\text {tot }}$ is the available power for each node of the network. Each transmitting node set its transmission parameters (TPs), i.e., the transmission mode $(\mathrm{TM}) \phi_{l} \triangleq\left\{\mathbf{m}_{l}, r_{l}\right\} \in \mathcal{D}_{\mathrm{m}}^{N} \times \mathcal{D}_{\mathrm{r}}$ and $\mathbf{p}_{l}$, by means of the selected RA strategy. After inverse fast Fourier transform (IFFT) with length $N_{\text {FFT }}$, cyclic prefix (CP) insertion, and digital-toanalog conversion, the multicarrier signal is transmitted over a frequency-selective multipath fading channel, which is assumed to be stationary for the whole packet transmission interval. At the receiver side, the estimation $\hat{\mathbf{H}}_{l} \triangleq\left[\hat{H}_{l, 1}, \cdots, \hat{H}_{l, N}\right]^{\mathrm{T}}$ of the actual channel $\mathbf{H}_{l} \triangleq\left[H_{l, 1}, \cdots, H_{l, N}\right]^{\mathrm{T}}$ allows the predicted SNR evaluation $\hat{\gamma}_{l, n} \triangleq \frac{\left|\hat{H}_{l, n}\right|^{2}}{\sigma_{w}^{2}}$ (see [6] for details), signal equalization and soft decoding of the received signal.

\section{Predicted GP Metric and Joint PS and RA Algorithms}

3.1 Predicted GP Metric for Multi-hop Networks with Imperfect and Outdated CSI.

Let us extend the evaluation of the predicted GP Metric (PGP) derived in [6] for dual-hop communications to a multi-hop scenario [6]. Given the generic path $\overline{\mathcal{P}}\left(\mathrm{SN}, \mathrm{R}_{i_{1}}, \cdots, \mathrm{R}_{i_{L}}, \mathrm{DN}\right)$ through $L, 1 \leq L \leq M, \mathrm{DF}$ $\mathrm{RNs}$, the probability of a packet error from SD to DN can be shown to be

$$
\begin{aligned}
& \Phi_{\mathrm{tot}}\left(r_{0}, \cdots, r_{L}, \gamma_{\mathrm{eff}, 0}, \cdots, \gamma_{\mathrm{eff}, L}\right) \\
& =\Phi_{0}\left(r_{0}, \gamma_{\mathrm{eff}, 0}\right)+\sum_{i=1}^{L} \prod_{k=0}^{i-1}\left[1-\Phi_{k}\left(r_{k}, \gamma_{\mathrm{eff}, k}\right)\right] \Phi_{i}\left(r_{i}, \gamma_{\mathrm{eff}, i}\right),
\end{aligned}
$$

where the PER of the $k$ th link $\Phi_{k}\left(r_{k}, \gamma_{\text {eff, } k}\right.$ is evaluated applying the $\kappa$ ESM method as a function of the equivalent SNR $\gamma_{\mathrm{eff}, k}$. Accordingly, the global PGP (G-PGP), i.,e., the PGP from SD to DN reads as

$$
\begin{array}{r}
\zeta_{\text {global }}\left(\phi_{0}, \cdots, \phi_{L}, \mathbf{p}_{0}, \cdots, \mathbf{p}_{L}\right) \\
=\frac{1}{B} \frac{N_{\mathrm{p}}\left[1-\Phi_{\mathrm{tot}}\left(r_{0}, \cdots, r_{L}, \gamma_{0, \mathrm{eff}}, \cdots, \gamma_{L, \mathrm{eff}}\right)\right]}{\sum_{j=0}^{L} T_{\mathrm{pkt}, j}\left(\phi_{j}\right)},
\end{array}
$$

where $\phi_{0}$ and $\mathbf{p}_{0}$ denotes the TPs of the SN for the link SN-R $i_{1}, \phi_{l}$ and $\mathbf{p}_{l}$ the TPs for the link $\mathrm{R}_{i_{l}}-\mathrm{R}_{i_{l+1}}$, and $\phi_{L}$ and $\mathbf{p}_{L}$ the TPs of $\mathrm{R}_{i_{L}}$ for the link $\mathrm{R}_{i_{L}}$-DN.

\subsection{Optimal Centralized Joint PS and RA Solution.}

Due to lack of space, we provide hereafter just a short outline of the optimal joint PS/RA technique which selects both the path and the TPs for the relevant nodes, yielding the best G-PGP between SN and DN (a detailed analytical description can be found in [6]). First, it is assumed here that the DN is a centralized processing unit which is responsible for all the computational complexity of the wireless network. So, the DN performs an exhaustive search over any possible path connecting SN and DN (possibly, via some RNs) and finds the path that maximizes the G-PGP (2) with a twostep procedure.

Step 1. Per-Path G-PGP Evaluation. For every path, the DN collects the predicted channel vectors of the relevant links and solves a RA problem to find the TPs providing the maximum G-PGP (2). The analysis has been carried out considering both uniform and non-uniform subcarrier bit-loading (BL) strategies at path level. The RA problem turns out a mixed integer $\mathrm{OP}$, which includes both variables belonging to a discrete finite-size set (e.g., TMs) and continuous-valued variables (e.g., the subcarrier power vector). Moreover, it can be shown that the problem can be sub-optimally, though efficiently, decoupled in two consecutive steps. Step 1.1. PA evaluation. Given a generic TM, the best PA vector is found by solving a convex OP, whose optimal solution can be found by means of the Lagrange multipliers method. The result turns out a water-filling-like solution which depends only on the BL vector, whereas it is independent of the coding rate. Step 1.2. TM evaluation. . Given the closed-form solution derived according to the procedure in previous Step 1.1., the best TM is selected by maximizing the G-PGP function (2). According to the above considerations, an analytical description of the PA problem is defined and solved as follows.

Uniform $B L(U-B L)$ solution. The $\mathrm{OP}$ is solved by means of an exhaustive search, with complexity $\mathcal{O}\left(\left(\left|\mathcal{D}_{\mathrm{r}}\right| \cdot\left|\mathcal{D}_{\mathrm{u}}\right|\right)^{L+1}\right)$.

Best $B L(B-B L)$ solution. A different number of bits can be loaded over each subcarrier based on the greedy algorithm, whose computational complexity is now $\mathcal{O}\left(\left(\left|\mathcal{D}_{\mathrm{r}}\right| \cdot\left|\mathcal{D}_{\mathrm{m}}\right| \cdot N \log N\right)^{L+1}\right)$ instead of $\mathcal{O}\left(\left(\left|\mathcal{D}_{\mathrm{r}}\right| \cdot\right.\right.$ $\left.\left.\left|\mathcal{D}_{\mathrm{m}}\right|^{N}\right)^{L+1}\right)$.

Step 2. Best Path Selection. The DN selects the path yielding the maximum G-PGP value and sends the optimal TPs the SN and the RNs belonging to the selected path. As explained above, the centralized PS and RA problems are solved in a exhaustive way, yielding an exponential growth in computational complexity on the DN, directly proportional to the number of RNs and making, therefore, this solution not scalable. Indeed, in a network with $M \mathrm{RNs}$ there exist $M ! /(M-L)$ ! different routes to connect SN and DN passing through $L \leq M \mathrm{RNs}$ and the cardinality of the set $\mathcal{G}_{\mathrm{S}}$ is $\sum_{l=0}^{M} M ! / l !$. Hence, an exhaustive search among all the possible candidate connections SN-DN requires a combinatorial complexity completely loaded over the DN. Moreover, the DN must receive the CSI from any link of the network and, once solved the centralized joint PS and RA problem, it must send the 
TPs to the SN and the RNs belonging to the final selected path. In this manner, a congestion of the feedback channel towards the DN is highly possible. For this reasons, in the next Subsection a distributed PS and RA approach is presented, which divides the computational complexity over all the nodes of the network. The centralized method will be therefore considered as a benchmark only.

\subsection{Distributed Joint PS and RA Strategy.}

The distributed joint PS and RA strategy is developed by formalizing the problem in the NFG framework.

Definition 1: i) $\mathcal{P}_{\mathrm{R}_{k}}\left(\mathrm{R}_{k}, \mathrm{R}_{i_{1}}, \cdots, \mathrm{R}_{i_{L}}, \mathrm{DN}\right)$ as the path connecting $\mathrm{R}_{k}$ to $\mathrm{DN}$ passing through the relays $\mathrm{R}_{i_{1}}, \ldots, \mathrm{R}_{i_{L}}$, with $0 \leq L \leq M-1, i_{1}, \cdots, i_{L} \in$ $\{1, \cdots, M\}$, with $i_{j} \neq i_{k}, \forall j \neq k \in\{1, \cdots, L\}$, and $\mathrm{R}_{i_{L}} \triangleq \mathrm{DN}$ if $L=0$, i.e., the direct link $\left.\mathrm{R}_{k^{-}} \mathrm{DN} ; i i\right)$ $l \in \mathcal{L}_{\text {relay }} \triangleq\{1, \cdots L\}$ as the index of the generic link, where $l=L$ concerns $\mathrm{R}_{i_{L}}$-DN and $l, 0<l<L, \mathrm{R}_{i_{l}}{ }^{-}$ $\mathrm{R}_{i_{l+1}}$.

Assumption: $i$ ) the network topology is described by a direct graph $G(\nu, \varepsilon)$, where $\nu=\{1, \cdots, M\}$ is the set of all vertices (RNs) and $\varepsilon$ is the set of all edges (links among RNs); $i i$ ) the $\mathrm{SN}$ is an external source of traffic, which accesses the network only when the topology of the latter is fixed. The approach allows to solve only one NFG problem, thus reducing both the waiting time of the SN to start its transmission and the signalling traffic. Hence, the RN-based NFG is defined by the following triplet:

- the set of RNs, i.e., the players, $\mathcal{R} \triangleq$ $\left\{\mathrm{R}_{1}, \cdots, \mathrm{R}_{M}\right\}$

- the set of strategies $\mathcal{S} \triangleq\left\{\mathcal{S}_{\mathrm{R}_{1}}, \cdots, \mathcal{S}_{\mathrm{R}_{M}}\right\}$, where for any $G, \mathcal{S}_{\mathrm{R}_{k}} \triangleq\left\{\mathcal{P}_{\mathrm{R}_{k}}, \phi_{\mathrm{R}_{k}}, \mathbf{p}_{\mathrm{R}_{k}}\right\}, \mathcal{P}_{\mathrm{R}_{k}} \in \mathcal{G}_{\mathrm{R}_{k}}$ being the set of all possible paths between $\mathrm{R}_{k}$ and $\mathrm{DN}$, $\phi_{\mathrm{R}_{k}} \triangleq\left(r_{\mathrm{R}_{k}}, \mathbf{m}_{\mathrm{R}_{k}}\right)$ being the pair code rate and BL vector $\mathbf{m}_{\mathrm{R}_{k}} \triangleq\left[m_{\mathrm{R}_{k}, 1}, \cdots, m_{\mathrm{R}_{k}, N}\right]^{\mathrm{T}}$ and $\mathbf{p}_{\mathrm{R}_{k}} \triangleq$ $\left[p_{\mathrm{R}_{k}, 1}, \cdots, p_{\mathrm{R}_{k}, N}\right]^{\mathrm{T}}$ being the $\mathrm{PA}$ vector, all the above TPs being referred to the first hop $s_{\mathrm{R}_{k}}$ of its path $\mathcal{P}_{\mathrm{R}_{k}}$;

- the set of the utility functions $\mathcal{U} \triangleq$ $\left\{\mathcal{U}_{\mathrm{R}_{1}}, \cdots, \mathcal{U}_{\mathrm{R}_{M}}\right\}, \quad$ where $\quad \mathcal{U}_{\mathrm{R}_{k}}\left(\mathcal{S}_{\mathrm{R}_{k}}, \mathcal{S}_{-\mathrm{R}_{k}}\right) \triangleq$ $\frac{1}{B} \frac{N_{\mathrm{p}}\left[1-\tilde{\Phi}_{\text {tot }}\left(\mathcal{S}_{\mathrm{R}_{k}}, \mathcal{S}_{-\mathrm{R}_{k}}\right)\right]}{\tilde{T}_{\mathrm{pkt}}^{\text {tot })}\left(\mathcal{S}_{\mathrm{R}_{k}}, \mathcal{S}_{-\mathrm{R}_{k}}\right)}, \quad \mathcal{S}_{-\mathrm{R}_{k}} \quad$ being the subset of all strategies of the $\mathrm{RNs}$ belonging to the path $\mathcal{P}_{\mathrm{R}_{k}}$ such that $\mathcal{S}_{-\mathrm{R}_{k}} \triangleq\left\{\mathcal{S} \backslash \mathcal{S}_{\mathrm{R}_{k}}: \mathrm{R}_{j} \in \mathcal{P}_{\mathrm{R}_{k}}, \forall j \in\{1, \cdots, M\}\right\}$, $\tilde{\Phi}_{\text {tot }}\left(\mathcal{S}_{\mathrm{R}_{k}}, \mathcal{S}_{-\mathrm{R}_{k}}\right)$ being the PER along the $\mathrm{R}_{k}$-DN link, depending on the PER over the first hop $\Phi_{s_{\mathrm{R}_{k}}}\left(r_{\mathrm{R}_{k}}, \gamma_{\mathrm{eff}, \mathrm{R}_{k}}\right)$ and the PER over the remaining part of the link $\Delta\left(\mathcal{S}_{-\mathrm{R}_{k}}\right) \triangleq \Phi_{1}\left(r_{1}, \gamma_{\mathrm{eff}, 1}\right)+$ $\sum_{i=2}^{L} \prod_{k=1}^{i-1}\left[1-\Phi_{k}\left(r_{k}, \gamma_{\mathrm{eff}, k}\right)\right] \Phi_{i}\left(r_{i}, \gamma_{\mathrm{eff}, i}\right), \quad$ and $\tilde{T}_{\mathrm{pkt}}^{\text {(tot) }}\left(\mathcal{S}_{\mathrm{R}_{k}}, \mathcal{S}_{-\mathrm{R}_{k}}\right) \triangleq T_{\mathrm{pkt}, s_{\mathrm{R}_{k}}}\left(\phi_{\mathrm{R}_{k}}\right)+\tilde{T}_{\mathrm{pkt}}\left(\mathcal{S}_{-\mathrm{R}_{k}}\right)$ being the packet transmission time, i.e., the one for the first hop and that due to the subsequent hops.

Definition 2: given a graph $G$ and the set strategies $\mathcal{S}$, let us define: $i$ ) a feasible strategy for the $\mathrm{RN} \mathrm{R}_{k}$ if $\mathcal{U}_{\mathrm{R}_{k}}\left(\mathcal{S}_{\mathrm{R}_{k}}, \mathcal{S}_{-\mathrm{R}_{k}}\right)>0$; ii) the set of the feasible strategies $\overline{\mathcal{S}}_{\mathrm{R}_{k}} \triangleq\left\{\mathcal{S}_{\mathrm{R}_{k}}: \mathcal{U}_{\mathrm{R}_{k}}\left(\mathcal{S}_{\mathrm{R}_{k}}, \mathcal{S}_{-\mathrm{R}_{k}}\right)>0\right\}$; iii) the best feasible strategy if $\mathcal{U}_{\mathrm{R}_{k}}\left(\mathcal{S}_{\mathrm{R}_{k}}^{*}, \mathcal{S}_{-\mathrm{R}_{k}}\right) \geqslant \mathcal{U}_{\mathrm{R}_{k}}\left(\mathcal{S}_{\mathrm{R}_{k}}, \mathcal{S}_{-\mathrm{R}_{k}}\right)$, $\left.\forall \mathcal{S}_{\mathrm{R}_{k}} \in \overline{\mathcal{S}}_{\mathrm{R}_{k}} ; i v\right) \mathcal{S}_{\mathrm{R}_{k}}^{*} \triangleq\left\{\mathcal{P}_{\mathrm{R}_{k}}^{*}, \phi_{\mathrm{R}_{k}}^{*}, \mathbf{p}_{\mathrm{R}_{k}}^{*}\right\}$ the best response (BR) of the NFG optimizing the utility function.

NFG Algorithm. The RN-based NFG is solved by the following two steps.

Step 1. The BR $\mathcal{S}_{\mathrm{R}_{k}}^{*}=\underset{\mathcal{S}_{\mathrm{R}_{k}} \in \overline{\mathcal{S}}_{\mathrm{R}_{k}}}{\operatorname{argmax}}\left\{\mathcal{U}_{\mathrm{R}_{k}}\left(\mathcal{S}_{\mathrm{R}_{k}}, \mathcal{S}_{-\mathrm{R}_{k}}\right)\right\}$ problem is iteratively solved for each $\mathrm{R}_{k}$ (thus obtaining the graph $G(\nu, \varepsilon)$ ), where each iteration consists of three consecutive tasks. Due to the exhaustive search (ES) performed by each $\mathrm{R}_{k}$ over $\mathcal{G}_{\mathrm{R}_{k}}$ to find the $\mathrm{BR}$, the proposed RN-based NFG will be labeled as distributed with ES (D-ES) algorithm.

1. Each $\mathrm{RN} \mathrm{R}_{k}$ gets $\overline{\mathcal{S}}_{\mathrm{R}_{k}}$, given $\overline{\mathcal{S}}_{-\mathrm{R}_{k}}$, $\forall k \in\{1, \cdots, M\}$, by evaluating its TPs over the first hop $s_{\mathrm{R}_{k}} \forall \mathcal{P}_{\mathrm{R}_{k}} \in \mathcal{G}_{\mathrm{R}_{k}}$ and collecting the sets $\mathcal{S}_{\mathrm{R}_{k}}$ such that $\mathcal{U}_{\mathrm{R}_{k}}\left(\mathcal{S}_{\mathrm{R}_{k}}, \mathcal{S}_{-\mathrm{R}_{k}}\right)>0$. Hence, given a generic path $\overline{\mathcal{P}}_{\mathrm{R}_{k}}\left(\mathrm{R}_{k}, \mathrm{R}_{i_{1}}, \cdots, \mathrm{R}_{i_{L}}, \mathrm{DN}\right) \in \mathcal{G}_{\mathrm{R}_{k}}$ and the set $\mathcal{S}_{-\mathrm{R}_{k}}$ of the other RNs belonging to $\overline{\mathcal{P}}_{\mathrm{R}_{k}},\left(\phi_{\mathrm{R}_{k}}^{*}, \mathbf{p}_{\mathrm{R}_{k}}^{*}\right)$ is found by evaluating $\arg \max \left\{\mathcal{U}_{\mathrm{R}_{k}}\left(\overline{\mathcal{P}}_{\mathrm{R}_{k}}, \phi_{\mathrm{R}_{k}}, \mathbf{p}_{\mathrm{R}_{k}}, \mathcal{S}_{-\mathrm{R}_{k}}\right)\right\} \quad$ s.t. $\phi_{\mathrm{R}_{k}}, \mathbf{p}_{\mathrm{R}_{k}} \geq 0$

$\sum_{n=1}^{N} p_{\mathrm{R}_{k}, n} \leq P_{\text {tot }}$ and $\mathcal{D}_{\mathrm{m}}^{N} \times \mathcal{D}_{\mathrm{r}}$, thus obtaining a closed-form water-filling-like solution for the PA vector as a function of the $\mathrm{BL}$ vector. Further, the best $\mathrm{TM} \phi_{\mathrm{R}_{k}}^{*}$ is found by solving $\underset{\phi_{\mathrm{R}_{k}}}{\arg \max }\left\{\mathcal{U}_{\mathrm{R}_{k}}\left(\overline{\mathcal{P}}_{\mathrm{R}_{k}}, \phi_{\mathrm{R}_{k}}, \mathbf{p}_{\mathrm{R}_{k}}^{*}\left(\mathbf{m}_{\mathrm{R}_{k}}\right), \mathcal{S}_{-\mathrm{R}_{k}}\right)\right\} \quad$ s.t. $\mathcal{D}_{\mathrm{m}}^{N} \times \mathcal{D}_{\mathrm{r}}$. Differently from the centralized algorithm in Sect. 3.1, it is worth noting that here the optimization variables are $\phi_{\mathrm{R}_{k}}$ and $\mathbf{p}_{\mathrm{R}_{k}}$ and $\phi_{\mathrm{R}_{k}}$ only, respectively, with a considerable reduction of the computational load.

2. The BR $\mathcal{S}_{\mathrm{R}_{k}} \in \overline{\mathcal{S}}_{\mathrm{R}_{k}}, \forall k \in\{1, \cdots, M\}$, is selected and sent to the DN.

3. At the end of the iteration, the DN broadcasts the selected BRs to all the RNs.

The iterations end when an equilibrium point is obtained, the Nash network, i.e., when there is no incentive for any RN to change its strategy to improve its utility function.

Step 2. Once a final network topology $G$ is built by the RNs during the Step 1, the SN can access the network and perform its PS and RA strategy by selecting the final path $\mathcal{P}_{\mathrm{S}}^{*}$ and best $\mathrm{TM} \phi_{0}^{*}$ and $\mathrm{PA}$ vector $\mathbf{p}_{0}^{*}$, corresponding to the connection $\mathrm{SN}-\mathrm{DN} \mathcal{P}_{\mathrm{S}}^{*}$ that maximizes the G-PGP. This is achieved by solving an optimization problem similar to those solved by the RNs at each iteration of the NFG of Step 1. 
3.4. Distributed Joint PS and RA Algorithm with Reduced Computational Complexity.

According to the D-ES of Sect. 3.3, a RN $\mathrm{R}_{k}$ iteratively builds the set $\overline{\mathcal{S}}_{\mathrm{R}_{k}}$ by performing an ES over all the possible paths (tough only the first hop and not the overall path is considered), and for each of them calculates the TPs, thus increasing the computational load for each node. Such a drawback can be skipped by applying to the D-ES the modified Bellman-Ford (MBF) algorithm [7], that gives the shortest paths in a graph $G$ for each possible hop count. The sub-optimal yet competitive modified algorithm we obtain, labelled as distributed with $\mathrm{BF}$ (D-BF), notably shows a polynomial, rather than combinatorial, complexity in the number $M$ of RNs.

\section{Numerical results and Conclusions}

The performance of the algorithms is expressed by the averaged GP (AGP), which is obtained by averaging the number of bits $/ \mathrm{s} / \mathrm{Hz}$ correctly received over $N_{\text {pkt }}$ transmitted packets. A multipath channel and a modified COST231 Hata path loss (PL) are implemented, considering different channel realizations with a Doppler frequency $f_{\mathrm{d}}$ for each transmitted packet. The setup consists in $N_{\text {pkt }}=1000$ transmitted packets, $N_{\mathrm{p}}=1024$ information bits, $N_{\mathrm{CRC}}=32$ CRC bits, $N=1320$ subcarriers, FFT size equal to 2048, bandwidth $B=20 \mathrm{MHz}$, Doppler frequency $f_{\mathrm{d}}=144 \mathrm{~Hz}$, bit set $\mathcal{D}_{\mathrm{m}}=\{2,4,6\}$, coding rate set $\mathcal{D}_{\mathrm{r}}=\{1 / 2,2 / 3,3 / 4,5 / 6\}$, and in-band noise power $P_{\text {noise }}=-100 \mathrm{dBm}$.

Fig. 1 illustrates the network scenario wherein the SN moves along the solid line, with $M=6$ and $d_{\min }=80$ $\mathrm{m}$. Figure 2 presents a comparison of the GP performance vs. SN-DN separation for D-ES and D-BF algorithms, with the optimal centralized algorithm and the direct SN-DN link solutions as benchmarks. We remark that: 1) performance loss with respect to the centralized solution is negligible when either D-ES or D-BF algorithms are used; 2) the performance are essentially the same between D-ES and D-BF, confirming the effectiveness of the uniform PA approximation for the latter approach.

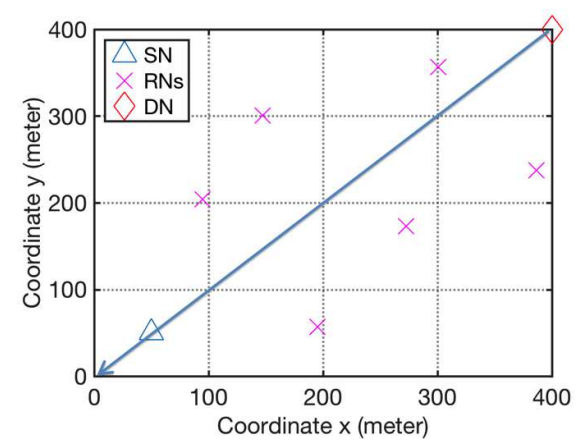

Figure 1. Network Scenario

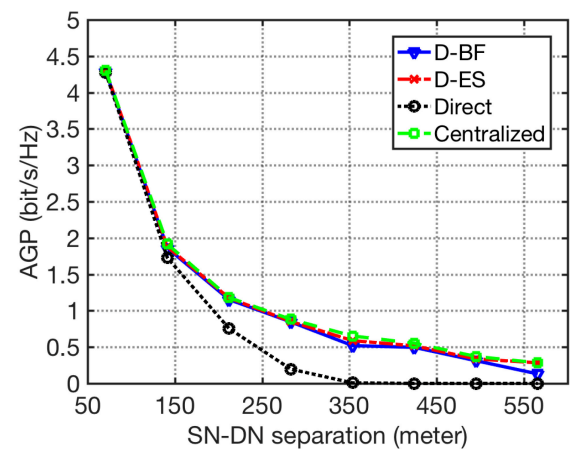

Figure 2. AGP vs. SN-DN separation with OPA, U-BL, $P_{\text {tot }}=25 \mathrm{dBm}, \Lambda=3$.

Concluding, this work proposed a novel distributed joint PS/RA strategy in multi-hop DF BIC-OFDM relay networks under imperfect and outdated CSI based on the modified Bellman-Ford algorithm. Simulation results confirm the effectiveness of the proposed distributed strategies, whose performance are close to the centralized case, but offering a great saving in signaling and computational complexity.

\section{Acknowledgement}

This work has been partially supported by the University of Pisa under the PRA 2018-2019 Research Project CONCEPT-COmmunication and Networking for vehicular CybEr-Physical sysTems.

\section{References}

[1] J. Boyer, D. D. Falconer and H. Yanikomeroglu, "Multihop diversity in wireless relaying channels," in IEEE Transactions on Communications, vol. 52, no. 10, pp. 1820-1830, Oct. 2004.

[2] R. Pabst et al., "Relay-based deployment concepts for wireless and mobile broadband radio," in IEEE Communications Magazine, vol. 42, no. 9, pp. 80-89, Sept. 2004.

[3] G. Demange and M. Wooders, Group Formation in Economics: Networks, Clubs and Coalitions. Cambridge Univ., 2005.

[4] W. Li, X. Cheng, T. Jing and X. Xing, "Cooperative multihop relaying via network formation games in cognitive radio networks," IEEE INFOCOM, pp. 971-979, 2013.

[5] W. Saad, Z. Han, T. Basar, M. Debbah and A. Hjorungnes, "Network Formation Games Among Relay Stations in Next Generation Wireless Networks," in IEEE Transactions on Communications, vol. 59, no. 9, pp. 2528-2542, September 2011.

[6] R. Andreotti, V. Lottici and Filippo Giannetti, "Results on Distributed Radio Resource Allocation for Multicarrier Relaying Networks", Tech. Report, Dept. Inf. Eng., University of Pisa, Pisa, Apr. 2019.

[7] R. Guerin, A. Orda, "Computing shortest paths for any number of hops," IEEE Trans. on Networking, vol.10, no.5, 2002 . 